\title{
LOW-ACTIVITY NUCLEI IN SPIRAL GALAXIES
}

\author{
G. Fabbiano \\ Harvard-Smithsonian Center for Astrophysics \\ Cambridge, Massachusetts, USA.
}

Two types of active nuclei have been observed in normal spiral galaxies in X-rays with the Einstein Observatory: low-activity AGN, and starburst regions. The prototype of the first kind is the nucleus of M81 (Elvis and Van Speybroeck 1982; Fabbiano 1988a), but similar nuclei might also be those of M33 and NGC 1313. Soft X-ray spectra of these nuclei suggest relatively steep soft X-ray components (Trinchieri, Fabbiano and Peres 1988; Fabbiano and Trinchieri 1987; Fabbiano 1988a), reminiscent of those observed in QSOs by Bechtold et al (1987) and Wilkes and Elvis (1987). In M81, in particular, this soft component might supply enough photons to explain the optical line spectrum. If this soft $\mathrm{X}$-ray component originates from an accretion disk surrounding a central black hole, the mass of the latter is likely to be smaller than $10^{4-5}$ solar masses.

Plumes of extended X-ray emission have been found associated with the starburst nuclei of NGC 253 and M82 (Fabbiano and Trinchieri 1984; Watson, Stanger and Griffiths 1984), suggesting nuclear gaseous outflows. Subsequent optical work by many authors has confirmed this view (e.g. Heckman, Armus and Miley 1987). A recent work on lowresolution X-ray images of these two galaxies suggest that this gaseous component extends at a significant distance from the galactic disks (Fabbiano 1988b). In M82, in particular, there is evidence of a gaseous halo, not bound to the system, extending as far as 9' from the nucleus along the minor axis. Very recently, a systematic analysis of X-ray images of spiral galaxies has revealed another such nucleus, in the edge-on galaxy NGC 3628. Follow up CCD imaging and optical spectroscopy by Heckman and Keel is confirming the presence of a nuclear outflow.

\section{REFERENCES}

Bechtold , J., Czerny, B., Elvis, M., Fabbiano, G., and Green, R.F. 1987, Ap. J., 314, 699.

Elvis , M. and Van Speybroeck, L. 1982, Ap. J. Letters, 257, L51.

Fabbiano , G. 1988a, Ap. J., 325, 544.

Fabbiano , G. 1988b, Ap. J., 330, 672.

Fabbiano , G. and Trinchieri, G. 1987, Ap. J., 315, 46.

Fabbiano , G. and Trinchieri, G. 1984, Ap. J., 286, 491.

Heckman , T.M., Armus, L., and Miley, G. 1987, A.J., 92, 276.

Trinchieri , G., Fabbiano, G., and Peres, G. 1988, Ap. J., 325, 531.

Watson , M.G., Stanger, V., and Griffiths, R.E. 1984, Ap. J., 286, 144.

Wilkes , B.J: and Elvis, M. 1987, Ap. J., 323, 243. 